\title{
Comunicação, cultura e mundo da vida: as contribuições de Jürgen Habermas e Alfred Schutz ${ }^{1}$
}

\author{
Michael Manfred Hankel \\ https://orcid.org/0000-0001-6102-7328 \\ I - Universidade Federal do Rio Grande do Norte. \\ Natal (RN), Brasil.
}

Resumo: Este artigo apresenta o conceito de mundo da vida e a sua contribuição-chave para uma teoria da cultura e da comunicação. Inicialmente desenvolvido pelo filósofo Edmund Husserl, o conceito foi aprimorado pelo filósofo e sociólogo austro-americano Alfred Schutz e adotado mais tarde por teorias consagradas, como as de Peter Berger e Thomas Luckmann no projeto de um construtivismo social, assim como por Jürgen Habermas no agir comunicativo como fundamento da razão, o que destaca a importância de tal empreendimento. $\mathrm{O}$ texto conceitua o mundo da vida como interface entre a teoria da comunicação e da cultura.

Palavras-Chave: mundo da vida; Jürgen Habermas; Alfred Schutz; comunicação e cultura.

\begin{abstract}
Communication, Culture and Lifeworld: The contributions of Jürgen Habermas and Alfred Schutz - This paper portrays the concept of lifeworld in the light of its contribution to a theory of culture and communication. Initially developed by the philosopher Edmund Husserl, the concept was refined by the austro-american philosopher and sociologist Alfred Schutz. Later on, the concept was employed by well-established theories like the social constructivism of Peter Berger and Thomas Luckmann, as well as Jürgen Habermas' theory of communicative action as a basis of rationality, which highlights the importance of this undertaking. The concept of the
\end{abstract}


lifeworld is, along these lines, conceived as an interface between a theory of communication and culture.

Keywords: lifeworld; Jürgen Habermas; Alfred Schutz; communication and culture.

\section{Introdução}

O conceito de mundo da vida a ser tratado no presente artigo foi inicial e filosoficamente cunhado pelo filósofo Edmund Husserl (1859-1938) no âmbito da sua fenomenologia. Na sequência, foi aperfeiçoado de uma forma significativa por seu discípulo, o também filósofo fenomenológico e sociólogo Alfred Schutz (1899-1959).

A obra de Schutz, austríaco nascido em Viena, apesar de escrita na sua maior parte em inglês (o idioma adquirido após sua emigração para os Estados Unidos) e não em alemão (sua língua materna), é pouco conhecida no Brasil ${ }^{2}$. Sem dúvida, contribui para tal desconhecimento a falta de tradução da sua obra para o português (a única edição disponível em português, Schutz (1979) infelizmente não chega à altura necessária para fins de pesquisa). Entretanto, a língua, tantas vezes um obstáculo subestimado para o acesso a um pensador, neste caso de uma obra em inglês não é um fator que impeça a recepção desse autor. Também não faltam referências para acusar a importância de Schutz. Vamos listar como a primeira a obra seminal de Peter Berger e Thomas Luckmann (2015), A construção social da realidade. Esta, uma das publicações mais influentes da sociologia e considerada pela Associação Americana de Sociologia um dos dez livros mais importantes dessa disciplina, também no Brasil não é somente um clássico da sociologia - atualmente disponível na $23^{a}$ edição — , mas também leitura obrigatória em muitas outras áreas das ciências sociais. Berger e Luckmann escrevem no prefácio desse livro célebre: “Em várias partes deste tratado

2 Aaustríaco, Schutz escreveu, no início de sua carreira, seu primeiro livro em alemão. Após sua emigração, em 1939, para os Estados Unidos, onde atuou em Nova York na New School for Social Research, sua obra foi publicada em inglês. Assim, se a consulta deveria ser da versão original da obra, tem que ser em alemão para a primeira fase, enquanto os Collected Papers foram originalmente produzidos em inglês. Hoje, época em que o inglês predomina como língua franca do mundo globalizado, o alemão não é mais um idioma geralmente dominado. Isso pode servir de consolo para quem não domina este idioma, já que o inglês é suficiente para conhecer essa obra adequadamente. O nome de Schutz, diante dessa história, se escreveu inicialmente com trema ("Schütz"), o que vale para as publicações dessa fase. Depois, nos Estados Unidos, o trema foi eliminado e seu nome virou "Schutz". Hoje usa-se a versão em inglês. Entretanto, as obras publicadas na versão alemã (com "Schütz") seguem a forma original. Também em português escreve-se, como é de costume no mundo anglo-saxônico, sem o trema. 
ficará clara a dívida que temos com o falecido Alfred Schutz. Gostaríamos, porém, de reconhecer aqui a influência do ensino e das obras de Schutz em nosso pensamento" (BERGER; LUCKMANN, 2015, p. 6).

Consequentemente, no capítulo em que Berger e Luckmann desenvolvem, como eles mesmos alegam, seus conceitos-chave (BERGER; LUCKMANN, 2015, p. 5), lê-se:

Esta secção inteira de nosso tratado é baseada no livro de Alfred Schutz e Thomas Luckmann, Die Strukturen der Lebenswelt [As Estruturas do Mundo da Vida], agora preparada para publicação. Em vista disto, abstemo-nos de fornecer referências individuais às passagens da obra publicada de Schutz, onde os mesmos problemas são discutidos (BERGER; LUCKMANN 2015, p. 36).

Mais uma vez, se faz necessário frisar essa homenagem: “Devemos a compreensão fundamental da necessidade desta redefinição [da sociologia do conhecimento] a Alfred Schutz. Em toda sua obra, como filósofo e sociólogo, Schutz concentrou-se sobre a estrutura do mundo do sentido comum da vida cotidiana" (2015, p. 30, itálico nosso), sendo o termo Lebenswelt aqui traduzido na expressão em destaque. O termo Lebenswelt, mundo da vida, em outras oportunidades no livro é apresentado em alemão, sem tradução, enquanto Berger e Luckmann preferem utilizar a expressão "vida cotidiana" (Alltagswelt) ao invés de Lebenswelt ("vida cotidiana", Alltagswelt, em alemão, corresponde em inglês, "the world of everyday life"). Essa referência por si só deveria ser suficiente para legitimar um interesse no conhecimento maior da obra de Schutz e do conceito de mundo da vida.

Outra referência de importância significativa é a de Jürgen Habermas e a sua obra principal Teoria do agir comunicativo (HABERMAS, 2012). Neste livro, contribuição essencial para o pensamento comunicacional do século $X X, 0$ autor desenvolve uma teoria social baseada em dois pilares: o mundo da vida e o sistema (HABERMAS, 2012, II, p. 216). Para "esclarecer melhor o conceito 'mundo da vida', central à teoria da comunicação" (ibidem, p. 231-232), ele se refere explicitamente a Schutz. Alega o filósofo frankfurtiano: "Refiro-me aos manuscritos póstumos de Alfred Schütz sobre As estruturas do mundo da vida, organizados e editados por Thomas Luckmann" (ibidem, p. 232). Para o autor, o pano de fundo de qualquer manifestação comunicativa "é constituído por definições da situação" (ibidem, p. 223), e uma situação, que sempre é envolvida "por um horizonte que se desloca junto com o tema", "constitui o 
recorte de um contexto de referências do mundo da vida, enfatizado por temas e articulado por objetos e planos de ação" (ibidem p. 225). Em relação a tais contextos, Habermas ainda enfatiza que "Alfred Schütz descreveu e ilustrou inúmeras vezes tais divisões sociais, espaciais e temporais do mundo da vida cotidiana" (ibidem). A partir de outra abordagem, encontramos uma valorização semelhante de Schutz e seu conceito de mundo da vida.

A importância de Schutz para a área da comunicação é destacada também por Adriano Duarte Rodrigues, como escreve no seu prefácio para o Livro Compós de 2012, Mediação \& Midiatização (JANOTTI JUNIOR et al., 2012). A fenomenologia de Schutz integra assim, para o comunicólogo lisboeta, a sua lista dos textos "fundacionais" (RODRIGUES, 2012, p. 14), entre outros como os da psicologia social de George Herbert Mead e do pragmatismo de William James que, por sua vez, deixaram suas marcas na obra de Schutz.

Schutz, por sua vez, desenvolve o seu pensamento basicamente a partir de Max Weber e Edmund Husserl, sendo o primeiro, Weber, considerado o fundador das ciências sociais, e o segundo, Husserl, o da fenomenologia. Ambos são abordagens de ordem paradigmática para o século $X X$, sem o qual o pensamento nas ciências humanas não seria o que é. No Brasil, Max Weber também é considerado uma referência importante e autor de algumas das obras mais impactantes do século XX. Husserl, que escreveu em alemão, certamente mais uma vez por motivos linguísticos, não é tão lido, porém a fenomenologia, seu projeto filosófico principal, não é menos importante para as ciências humanas. Ou seja, não somente o próprio Schutz, mas também as suas referências são de alta relevância. E o mundo da vida é um conceito-chave da abordagem de Schutz.

\section{A reinterpretação do mundo da vida de Schutz em Habermas}

A seguir, vamos primeiramente analisar qual é o motivo que faz Habermas integrar o conceito de mundo da vida de Schutz na sua teoria do agir comunicativo e como ele contribui na construção da teoria. A resposta, obviamente, deve elucidar o valor do conceito.

Lembramos que o tema de Habermas é a "racionalidade de opiniões e ações" que, por sua vez, faz parte do contexto maior da "razão", o "tema fundamental da filosofia" (HABERMAS, 2012, I, p. 19). Entretanto, como fracassaram "todas as tentativas de fundamentação última [...] da filosofia original" (ibidem, 
p. 21), o autor busca outro caminho, o que o leva para as ciências sociais. Entre estas, "é a sociologia que está mais intimamente ligada, em seus conceitos fundamentais, à problemática da racionalidade" (ibidem, p. 23), e "que revela uma disposição especial a assumir o problema da racionalidade" (ibidem, p. 27). Isso leva o autor a uma determinação conceitual provisória, que articula o tema da racionalidade na base da sociologia e, consequentemente, em termos do mundo da vida. Segundo esta, o mundo exige como característica uma objetividade, precondição, além do saber proposicional, ponto de partida para uma análise da racionalidade. E vale para essa análise que:

O mundo só conquista objetividade ao tornar-se válido enquanto mundo único para uma comunidade de sujeitos capazes de agir e utilizar a linguagem. O conceito abstrato de mundo é condição necessária para que os sujeitos que agem comunicativamente possam chegar a um entendimento mútuo sobre o que acontece no mundo ou sobre o que se deve fazer nele. Com essa prática comunicativa, eles ao mesmo tempo se asseguram do contexto vital que têm em comum, isto é, de seu mundo da vida intersubjetivamente partilhado (ibidem, p. 40, itálico no original).

A referência de Habermas para a introdução desse "mundo compartilhado intersubjetivamente (o mundo da vida) (commonly shared world (Lebenswelt))", a qual remetem as "condições de validade das exteriorizações simbólicas" de "um saber fundamental partilhado intersubjetivamente pela comunidade de comunicação", é Schutz (além de Melvin Pollner, se referindo a Schutz) (ibidem, p. 40-41).

Ao elaborar "esse conceito de racionalidade comunicativa mais abrangente, desenvolvido a partir de um enfoque fenomenológico" (ibidem, p. 42), e "esclarecer o difícil conceito de mundo da vida racionalizado", Habermas quer "tomar como ponto de partida o conceito de racionalidade comunicativa e, então, investigar as estruturas do mundo da vida que possibilitam a indivíduos e a grupos orientações para a ação" (ibidem, p. 93). Entretanto, como "o conceito de mundo da vida é complexo demais para que eu possa explicá-lo satisfatoriamente no âmbito da introdução", essa explicação está prevista mais adiante no capítulo "O conceito 'mundo da vida' e o idealismo da sociologia hermenêutica", "vol. 2, pp. 218Ss" (ibidem, p. 93). Não obstante, já está assegurado desde o início da obra o lugar central do conceito de mundo da vida na teoria habermasiana, o que, pois, o leva a: 
[...] introduzir o conceito de mundo da vida, inicialmente como correlato dos processos de entendimento. Sujeitos que agem comunicativamente buscam sempre o entendimento no horizonte de um mundo da vida. O mundo da vida deles constitui-se de convicções subjacentes mais ou menos difusas e sempre isentas de problemas. Esse pano de fundo ligado ao mundo da vida serve como fonte de definições situacionais que podem ser pressupostas pelos partícipes como se fossem isentas de problemas. Em suas realizações interpretativas, os envolvidos em uma comunidade de comunicação estabelecem limites entre o mundo objetivo único e seu mundo social intersubjetivamente partilhado, de um lado, e os mundos subjetivos de indivíduos e de (outras) coletividades (ibidem, p. 138).

Ainda, esse "mundo da vida acumula o trabalho interpretativo prestado pelas gerações precedentes; ele é o contrapeso conservador que se opõe ao risco de dissenso, que surge com todo processo atual de entendimento" (ibidem, p. 139). Este é o pano de fundo da "racionalização do mundo da vida" (ibidem).

Dando sequência ao tema do compreender, Habermas destaca: "No contexto da sociologia alemã dos anos 1920, A. Schütz foi quem refletiu da maneira mais consequente sobre as implicações do acesso hermenêutico à realidade simbolicamente pré-estruturada" (ibidem, p. 228). O básico para essa teoria de ação é a decisão metodológica "de descrever a realidade social de tal modo que ela seja concebida como uma construção do mundo cotidiano surgida das realizações interpretativas dos que estão imediatamente envolvidos" (ibidem). Este princípio que orienta a compreensão do mundo social está também no foco da etnometodologia, tratada por Habermas em seguida e que, por sua vez, remete a Schutz (ibidem, p. 232).

A incorporação definitiva do conceito de Schutz por Habermas é, como anunciado, realizada na "Segunda consideração intermediária: mundo da vida e sistema" (HABERMAS, 2012, II, p. 205-355). Neste capítulo, quase um livro inteiro, Habermas introduz "o conceito 'mundo da vida"', que "constitui um conceito complementar ao do agir comunicativo" (ibidem, p. 218); entretanto, como "o alcance do conceito 'mundo da vida', que se oferece na perspectiva conceitual do agir orientado pelo entendimento, é limitado", necessitando, assim, do complemento do conceito de sistema, o autor propõe "um conceito de sociedade capaz de englobar o mundo da vida e o sistema" (ibidem, p. 216, itálico no original). Evidentemente, não dispomos de espaço para detalhar a argumentação na íntegra, mas apenas os pontos principais. 
A função principal do mundo da vida é gerenciar "estruturas tidas como invariantes" (ibidem, p. 219), ou seja, providenciar uma estabilidade perante a transformação e variabilidade do fluxo da vida. Desta forma, o mundo da vida serve como "pano de fundo de uma manifestação comunicativa", que "é constituído por definições da situação, as quais, tendo em vista a atual necessidade de entendimento, precisam se encaixar de certa forma" (ibidem, p. 223). Tal processo possibilita atribuir "certos conteúdos a determinados mundos" e assim, com "cada nova definição comum da situação, os atores determinam os limites entre a natureza exterior, a sociedade e a natureza interior", renovando, entretanto, "as diferenças entre eles", "perante o mundo exterior e perante seus respectivos mundos interiores" (ibidem). Mantido comunicativamente, incluindo a opção de redefinições, se estabelece um mundo objetivo, um mundo social e um mundo subjetivo próprio de cada um como "pano de fundo" com uma "validade intersubjetiva" (ibidem, p. 224-225). ${ }^{3}$

Entretanto, o apoio de Habermas em Husserl e Schutz não é incondicional. Ele rejeita os "conceitos da filosofia da consciência que Husserl utiliza para tratar da problemática do mundo da vida" (ibidem, p. 228), uma herança a ser encontrada também em Schutz. "Schütz manteve o princípio da fenomenologia transcendental [o método desenvolvido por Husserl] apesar das ressalvas feitas" (ibidem, p. 262), e por este motivo Habermas faz uma releitura do conceito de mundo da vida em termos da teoria da comunicação (ibidem). Destarte, "o problema que Husserl não conseguiu resolver nas Meditações cartesianas" foi, "a saber, o da produção monadológica da intersubjetividade do mundo da vida" (ibidem, p. 237, itálico no original). E Habermas critica: "Schütz e Luckmann não abandonam o modelo da filosofia da consciência", com a consequência de que: "Seguindo Husserl, eles tomam como ponto de partida a consciência 'egológica'" (ibidem, p. 236). Apesar da "influência do pragmatismo americano, em especial o de Mead", sublinhado por Luckmann, Habermas, que por sua vez se apoia em Mead para justificar o paradigma comunicacional, critica:

Schütz não se converte a uma teoria de comunicação, pois continua apegado ao método intuitivo de Husserl, assumindo, também, a arquitetônica da fenomenolo-

3 Estas condições de troca racional de argumentos podem ser alteradas pelo poder e pela ideologia, que mudam o pano de fundo ao qual se remetem os interlocutores, e levam à uma comunicação sistematicamente distorcida (como apontado por Bohmann, 2000, e Gross, 2010). Este apontamento foi feito por um dos avaliadores desse artigo, com agradecimentos. 
gia transcendental, o que o leva a interpretar seu próprio empreendimento como uma ontologia regional da sociedade. Isso explica porque Schütz e Luckmann não conseguem apreender as estruturas do mundo da vida diretamente das estruturas da intersubjetividade produzida de modo linguístico, sendo levados a buscá-las no reflexo da vivência subjetiva de atores solitários. No quadro da filosofia da consciência, o `sujeito que vivencia' continua sendo o ponto de referência derradeiro da análise (ibidem, p. 238-239).

Por isso, apesar "de um a priori social embutido na intersubjetividade do entendimento linguístico", Habermas, na sequência, continua criticando: "Em virtude das premissas da filosofia da consciência, nas quais se apoiam, Schütz e Luckmann subestimam o valor posicional da linguagem, especialmente o da mediação linguística da interação social" (ibidem, p. 240). Ainda, a abordagem de Husserl e Schütz, na tradição fenomenológica, leva a "um conceito de mundo da vida reduzido de modo culturalista" (ibidem, p. 254, itálico no original), crítica aplicada também a Berger e Luckmann no seu empreendimento de uma sociologia do saber e a "construção social da realidade" (ibidem). Dessa forma, "o conceito 'mundo da vida' reaparece numa versão institucionalista unilateral, comprimido numa teoria de socialização" (ibidem, p. 255, itálico no original). Ou seja, a realidade da sociedade em termos do mundo da vida não pode se limitar a uma interpretação cultural ou social, ela vai além disso e é moldada também por impactos sistêmicos. "De fato, porém, suas ações dirigidas a fins não se coordenam apenas mediante processos de entendimento, mas também por meio de contextos funcionais não visados por eles e em geral não percebidos no horizonte da prática cotidiana", sendo que: "Nas sociedades capitalistas, o mercado constitui o exemplo mais importante de uma regulação não normativa de contextos de cooperação" (ibidem, p. 272). Em outras palavras, a sociedade não pode ser reduzida a "um processo de socialização que se realiza por meio da consciência e da vontade de seus membros adultos" (ibidem, p. 271).

A centralidade do conceito de mundo da vida para Habermas mais uma vez se mostra na conclusão final, segundo o qual "a mediatização do mundo da vida se transforma em colonização", quando não há mais uma "reprodução simbólica do mundo da vida" (ibidem, p. 355, itálico no original). O conceito de agir comunicativo pressupõe "um medium linguístico" (2012, I, p. 182), uma "linguagem como um medium que transmite valores culturais e sustenta um consenso que simplesmente se reproduz com qualquer ato adicional 
de entendimento" (ibidem, p. 183) - vale lembrar que media significa para Habermas, além da linguagem, "meios de controle" ("Steuerungsmedien", em alemão), "como o dinheiro e o poder" (2012, II, p. 330), "(m)eios de comunicação não linguísticos" (ibidem, p. 333), que levam a uma "tecnicização do mundo da vida" (ibidem, p. 331, itálico no original). Uma estipulada "'mediatização' do mundo da vida", segundo o filósofo, exige uma diferenciação entre mundo da vida e sistemas de tal forma que estes conseguem influenciar o outro (ibidem, p. 336). O mundo da vida é quem sofre os efeitos da mediatização, e o sistema, que os causa, é cego por aquilo que faz:

\begin{abstract}
A "mediatização" do mundo da vida se realiza nele mesmo e com o auxílio de suas próprias estruturas; pois ela não faz parte dos processos disponíveis tematicamente no interior do mundo da vida, não podendo, por isso, ser extraída dos estoques da tradição, nem dos conteúdos da comunicação, ou do saber intuitivo dos membros. De outro lado, os fenômenos da interferência também não são acessíveis na perspectiva externa da teoria sistêmica. Eles não são percebidos na intuição ou na perspectiva interna do mundo da vida; no entanto, delineiam-se nas condições formais do agir comunicativo (ibidem, p. 336-337).
\end{abstract}

Dessa forma, Habermas modifica o conceito de mundo da vida pela inclusão de uma perspectiva da teoria da comunicação e do suplemento do sistema como segundo pilar da sociedade moderna.

\title{
O mundo da vida segundo Schutz
}

Schutz escreveu a sua obra magna tardia de referência, os dois volumes intitulados As Estruturas do Mundo da Vida, em inglês (The Structures of the LifeWorld), no final da década de 50. Ele faleceu antes da finalização do segundo volume, em 1959, sendo que seu aluno Thomas Luckmann finalizou a obra e, por isso, constam dois nomes como autores desta ${ }^{4}$. Assim, temos três autores envolvidos: Husserl, que elaborou o conceito, Schutz, que o aperfeiçoou em sua abordagem, não só na obra final, e Schutz e Luckmann, na finalização.

Os dois volumes de As Estruturas do Mundo da Vida, de 800 páginas de envergadura, se dividem em seis capítulos: 1) o mundo da vida cotidiana e a orientação (ou: atitude) natural; 2) a sedimentação (estrutura) do mundo da vida; 3) o saber (conhecimento) do mundo da vida; 4) conhecimento e sociedade; 
5) mundo da vida como campo da prática; 6) limitações de experiência e suas transcendências: comunicação no mundo da vida. Também neste caso, obviamente, não dispomos de espaço para detalhar a argumentação na sua íntegra, mas apenas os pontos principais. Contribui, para isso, que durante a produção desta obra maior, Schutz escreveu em 1957 um artigo em alemão "Estruturas do mundo da vida" ("Strukturen der Lebenswelt"), publicado primeiro com tradução em inglês em 1966 como "Some Structures of the LifeWorld". O texto, previsto para um congresso (Colloque Phénomenologique, em Pádua, em 1958, porém cancelado), foi republicado na versão original revisado (2003c). Conceitualmente este, obviamente escrito no contexto da obra maior, elabora os seguintes aspectos.

1. Constância. O mundo da vida é dado num modo de autoevidência e de forma não problemática. A sua estrutura, em conjunto com sua atitude (orientação) natural fornecida, possibilita experienciar a natureza, a cultura e a sociedade ${ }^{5}$. É produzida uma constância dela pela idealidade husserliana do 'e assim por diante', que cria a confiança, segundo o qual o mundo, da forma como é conhecido, vai continuar a existir dessa maneira, e o estoque de conhecimento (o meu e o socialmente compartilhado) manterá principalmente sua validade. Surge disso a suposição, que eu posso (futuramente) repetir minhas ações bem-sucedidas (do passado), criando a idealidade do 'eu posso sempre e de novo' (SCHUTZ, 2003c, p. 327). Assim, garante-se a constância da estrutura do mundo até uma nova ordem, assim como uma constância da validade de nossas experiências dele, e uma da nossa competência de agir nele.

2. Resolução de problemas. A autoevidência vale até uma nova ordem, quando expectativas "explodem". O que valeu antes não vale mais agora, ou o que sempre foi realizado não pode ser mais, e desta forma é que aparece um problema. Tais problemas teóricos, práticos ou emocionais aparecem diante de um pano de fundo de autoevidências. A resolução do problema se dá através de sua transformação em

\footnotetext{
50 texto em inglês só fala de cultura e sociedade ("culture and society", SCHUTZ, CP III, p. 116), omitindo a natureza do texto original (corretamente traduzido como "culture, nature and society"), o que demonstra mais uma vez a importância de desconfiar de traduções em contextos acadêmicos. Neste caso, o aquecimento global, por exemplo, enquanto fenômeno da natureza não fazia parte do mundo da vida que, na verdade, segundo o texto original, fazia. A "natureza", afinal, é um conceito cultural, como frisa Schutz: "Nature in this sense, as an element of the life-world, is thus a concept which has its place exclusively in the mental (geistig) sphere" (CP 1, p. 127, itálico no original).
} 
nova autoevidência e o fim desse processo é marcado pelo motivo pragmático da relevância.

3. Sedimentação. Longe de ser uma estrutura homogênea, o mundo da vida é uma sedimentação estruturada por divisões sociais, espaciais e temporais. Estas apresentam zonas com acessibilidade diferenciada, tendo, no seu centro, o mundo atualmente ao alcance (audível, visível, ou manipulável, seja diretamente ou por meio de instrumentos), além do mundo antigamente ao alcance (uma condição que atualmente não vale mais versus uma parte que é restaurável), e o mundo virtualmente acessível; todas essas zonas, por sua vez, com variações infinitas (ibidem, p. 329). Estes operam em dimensões de temporalidade objetiva, com correlatas subjetivas, voltado para o passado (chamado na terminologia fenomenológica de retention) ou o futuro (protention), memória e expectativas (em inglês: "retention and protention, recall and expectancy", SCHUTZ, CP III, p. 118). Em termos sociais, o mundo da vida é estruturado por relações sociais diferenciadas em um mundo de contato social direto (Umwelt, ao alcance ou ao redor atual), o mundo contemporâneo (o Mitwelt, dos contemporâneos, de alcance diferenciado), o mundo dos predecessores (Vorwelt, passado; seus integrantes podem nos influenciar, mas o contrário é impossível) e o mundo dos sucessores (Nachwelt, mundo futuro; nós podemos exercer impactos sobre seus integrantes; o contrário não procede. Contudo, eles ainda nem sabem da nossa existência).

4. Tipificações e simbolizações. Os esquemas de interpretação, que os atores sociais utilizam, são organizados em formas de tipificações e simbolizações, que por sua vez fazem parte do estoque de conhecimento do mundo da vida não questionado, dado de forma não problemática. Estes são de caráter intersubjetivo, ou seja, do grupo ao qual nós pertencemos, e é chamado de "cultura" (SCHUTZ, 2003c, p. 330).

5. Sociabilidade. A menor parte do estoque de conhecimento é individual, porém a maior parte dele é de natureza social e intersubjetiva, ou seja, ele depende da comunicação e é aprendido através de relações sociais de formas mais variadas. As lacunas do conhecimento, que é fragmentário e distribuído de forma desigual entre os sujeitos e grupos, são preenchidas socialmente. Problemas, que são 
interpretados à luz das suas tipicidades, são resolvidos por meios tipicamente aplicados para solucioná-los e rendem resultados que são tipicamente alcançados por estes meios. Essa tipicidade facilita o compartilhamento social.

6. Relevância. A seleção dos elementos do estoque de conhecimento é realizada de acordo com nosso interesse através da categoria fundamental de relevância, que define o que é considerado um problema isolado de um pano de fundo não problemático. Isso por sua vez define o nosso interesse, ou o que a psicologia tradicional chamou de "atenção". Não tendo acesso irrestrito à natureza, cultura e sociedade na sua totalidade, fazemos recortes delas, a partir de uma situação definida por nós. Qualquer situação é composta, de um lado, por um elemento ontológico (objetivo), que não está a disposição do ator (Schutz cita o exemplo de Bergson, segundo o qual para açucarar uma água é necessário esperar o açúcar se dissolver (ibidem, p. 332)); e de outro lado, por um elemento biográfico (subjetivo), com a liberdade de escolhas espontâneas. A doutrina da relevância é considerada "de importância fundamental para a teoria das ciências sociais" (ibidem, p. 341).

7. Linguagem. Os sistemas de relevância do mundo da vida se refletem na linguagem que armazena estas tipificações, como Schutz alega referindo-se a Wilhelm von Humboldt.

Não somente o vocabulário, mas também a estrutura sintática da linguagem corrente, denominado por Wilhelm von Humboldt como 'forma interna da linguagem', contém um sistema de tipificações e interpretações de relevâncias, que a comunidade linguística considera comprovado, não problemático e válido até ser revogado e, portanto, é passada para novos membros do grupo em processos de educação e aprendizagem (Ibidem, tradução nossa).

Habermas, no seu trato do mundo da vida, chega a constatar uma frase bem similar, também remetendo a Humboldt. Escreve o filósofo frankfurtiano:

Admitindo-se um nexo interno entre as estruturas do mundo da vida e as estruturas da imagem linguística do mundo - como é de praxe numa tradição que remonta a Humboldt -, a linguagem e a tradição cultural adquirem, de certo modo, uma posição transcendental em relação 
a tudo o que pode vir a ser componente de uma situação (HABERMAS, 2012, II, p. 228).

O que vale para a linguagem, vale também para os outros meios. A cultura, ou seja, "ferramentas, procedimentos, instituições sociais, costumes, rituais, sistemas simbólicos ou regras de conduta" (SCHUTZ, 2003c, p. 341-342, tradução nossa), disponibiliza uma orientação típica no mundo da vida e seu domínio. Todos estes elementos compõem o estoque de conhecimento do mundo da vida do grupo e possibilitam ao indivíduo a definição da sua situação e agir adequadamente. Constituem também o pano de fundo comum que possibilita a comunicação com os outros, os co-homens (ibidem, p. 342; em Habermas (2012, II, p. 241), este termo "co-homens" é utilizado no lugar de "próximos").

8. Comunicação. Schutz percebeu claramente a importância da comunicação para o homem e a sociedade, como destaca: “O mundo externo cotidiano é a realidade primorosa [...] porque [...] dentro desse recorte da realidade, e somente nele, podemos comunicar com nossos compatriotas [co-homens] e assim estabelecer um 'ambiente comunicativo comum' no sentido de Husserl" (SCHUTZ, CP I, p. 342, tradução nossa). No segundo volume de As Estruturas do Mundo da Vida, aquele que foi publicado após a crítica de Habermas sobre a falta de teoria da comunicação em Schutz, este último dedica um capítulo ao assunto "comunicação no mundo da vida" ("Verständigung in der Lebenswelt" (SCHUTZ, 1984, p. 201-212)). Como escreve Schutz no capítulo "as transcendências do mundo da vida através de signos e símbolos" (ibidem, p. 227), a comunicação somente se realiza através de signos (símbolos) (ibidem, p. 228). Ele ainda levanta a questão se, como defendido frequentemente, toda relação sígnica e simbólica envolve pelo menos três termos, dos quais um é o sujeito ou intérprete do signo, que pressupõe necessariamente uma inserção em processos de comunicação, ou se seria possível admitir a possibilidade de uma relação sígnica e simbólica no âmbito da vida psicológica ou mental de um indivíduo solitário (ibidem, p. 305). De qualquer forma, o fato de o mundo da vida ser essencialmente intersubjetivo implica a existência de co-homens, repletos de consciência, com os quais uma comunicação é possível, num contexto de uma estrutura da sociedade e cultura historicamente dada (ibidem, p. 317) 
Ou, repetindo isso em "Some Leading Concepts of Phenomenology":

Que contemporâneos existem, que homens agem sobre homens, que comunicação através de símbolos e signos é possível, que grupos sociais e instituições, sistemas jurídicos, econômicos e semelhantes são elementos integrais do nosso mundo da vida, que este mundo da vida tem sua própria história e relação específica com o tempo e o espaço - todas estas são noções que são explicitamente fundamentais para o trabalho de todos os cientistas sociais (SCHUTZ, CP 1, p. 116, tradução nossa).

Tal comunicação pressupõe que os esquemas de interpretação aplicados pelos dois participantes (falante e ouvinte, emissor e receptor) coincidem substancialmente (SCHUTZ, 1984, p. 326), constituindo um "universe of discourse" (em inglês) (ibidem, p. 327). Destarte, o instrumento por excelência ("kat exochen") da comunicação é a linguagem (ibidem, p. 388).

\section{Considerações finais}

Assim, nos parece que o conceito de mundo da vida, como o desenvolvido por Schutz e Habermas, é uma abordagem promissora para tratar o tema da comunicação e da cultura em sua complexidade no contexto da sociedade contemporânea. Em consonância com sua origem fenomenológica, induz a um conceito de realidade como esta se apresenta para o ator social, baseado na subjetividade da percepção, da interpretação (portanto, da hermenêutica) e sua inserção em grupos sociais, acompanhado por suas respectivas limitações, como expressado na crítica da redução culturalista. Historicamente, preparou o terreno para o paradigma do construtivismo social, que considera a realidade não como dada, mas construída em processos sociais. Aspectos sistêmicos de poder, de ideologia ou de política, entretanto, não estão no foco central dessa abordagem, que ainda favorece uma perspectiva micro em vez de macro.

Após uma certa parada, a recepção da abordagem schutziana ganhou novo fôlego, em 2009, com o lançamento da revista Schutzian Research ${ }^{6}$ e com o congresso bianual do International Alfred Schutz Circle for Phenomenology and Interpretive Social Science ${ }^{7}$. Ambos demonstram a fecundidade e a aplicabilidade das teorias de Schutz, seja no congresso que abordou as fake news em

6 Disponível em: <https://www.pdcnet.org/schutz/Schutzian-Research>. Acesso em: 24 abr. 2020.

7 Disponível em: <https://www.schutzcircle.org/>. Acesso em: 24 abr. 2020. 
2018 (BUECHNER et al., 2018, HANKE, 2018), ou no congresso de 2020 (agora postergado para 2021) sobre migração, imigração e refugiados, se valendo do trabalho seminal de Schutz, O Estrangeiro - Um ensaio em Psicologia Social (traduzido para o português (SCHUTZ, 2010)), que analisou os obstáculos que o mundo da vida representa para essa condição específica do estrangeiro. Essa retomada da abordagem schutziana pode permitir ampliar o debate a respeito da construção da realidade que, atualmente, por vezes, parece reduzida ao pano de fundo sociocultural ${ }^{8}$ e a um determinismo que o próprio Schutz não aderiu com sua visão mais complexa e diferenciada.

Michael Manfred Hanke é pesquisador da UFRN.

michaelhankebeaga@yahoo.com.br

\section{Referências}

BERGER, P. L.; LUCKMANN, T. A construção social da realidade: tratado de sociologia do conhecimento. Trad. de Floriano de Souza Fernandes. Petrópolis: Editora Vozes, 2015.

BOHMAN, J. When Water Chokes: ideology, communication, and practical rationality. In: Constellations 7 (3), 2000, p. 382-392.

BUECHNER, B.; VAN MIDDENDORP, S.; SPANN, R. Moral Injury on the Front Lines of Truth: Encounters with Liminal Experience and the Transformation of Meaning. In: Schutzian Research 10, 2018, p. 51-84.

GROSS, A. Systematically Distorted Communication: an impediment to social and political change. In: Informal Logic 30 (4), 2010, p. 335-360.

HABERMAS, J. Teoria do Agir Comunicativo. I: Racionalidade da ação e racionalização social. II: Sobre a crítica da razão funcionalista. Trad. de Flávio B. Siebeneichler. São Paulo: WMF Martins Fontes, 2012.

HANKE, M. Alfred Schütz: Einführung. Viena: Passagen Verlag, 2002. . Schutz' Semiotics and the Symbolic Construction of Reality. In: Schutzian Research 8, 2016, p. 103-120.

. Truth as Objectified Knowledge in In-Groups: Approaching Fake News within the Schutzian Framework. In: Schutzian Research 10, 2018, p. 141-153.

MÜLLER-DOHM, S. Jürgen Habermas. Eine Biographie. Berlim: Suhrkamp, 2014. 
RODRIGUES, A. D. Prefácio. In: Jeder Janotti Junior, Maria Ângela Mattos, Nilda Jacks (org.): Mediação \& Midiatização. Livro Compós 2012. Salvador: EDUFBA, 2012, p. 9-19.

SCHUTZ, A. Fenomenologia e Relações Sociais. Textos escolhidos de Alfred Schutz. Organização e introdução de Helmut R. WAGNER. Rio de Janeiro: Zahar, 1979.

Some Structures of the Life-World. In: Collected Papers III, 1970, p. 116-132.

Collected Papers. I. The Problem of Social Reality. The Hague: Martinus Nijhoff 1962. II. Studies in Social Theory. The Hague: Martinus Nijhoff 1964. III. Studies in Phenomenological Philosophy. The Hague: Martinus Nijhoff 1970. IV. Dordrecht: Kluwer 1996.

SCHÜTZ, A. Theorie der Lebenswelt 1. Die pragmatische Schichtung der Lebenswelt. Herausgegeben von Martin Endress und Ilja Srubar. Konstanz: UVK, 2003a. (ASW V.1)

Theorie der Lebenswelt 2. Die kommunikative Ordnung der Lebenswelt. Herausgegeben von Hubert Knoblauch, Ronald Kurt und Hans-Georg Soeffner. Konstanz: UVK 2003b.

Strukturen der Lebenswelt. In: Theorie der Lebenswelt 1, 2003c, p. 325-347.

. O Estrangeiro - Um ensaio em Psicologia Social. In: Revista Espaço Acadêmico 113, outubro de 2010, p. 117-129.

SCHUTZ, A.; LUCKMANN, T. Strukturen der Lebenswelt. Band 1. Frankfurt am Main: Suhrkamp, 1979.

Strukturen der Lebenswelt. Band 2. Frankfurt am Main: Suhrkamp, 1984.

Ensaio recebido em 07/01/2020 e aprovado em 30/05/2020. 